\title{
Norois
}

Environnement, aménagement, société

$214 \mid 2010 / 1$

Le Maroc en mutation

\section{Patrimoine et développement local à Fès : priorités, acteurs et échelles d'action}

cultural heritage and local development in Fez (Morocco): priorities, actors and action scales

\section{Brahim Akdim et Mohamed Laaouane}

\section{OpenEdition}

Journals

\section{Édition électronique}

URL : http://journals.openedition.org/norois/3083

DOI : $10.4000 /$ norois.3083

ISBN : 978-2-7535-1562-8

ISSN : $1760-8546$

Éditeur

Presses universitaires de Rennes

\section{Édition imprimée}

Date de publication : 20 mai 2010

Pagination : 9-21

ISBN : 978-2-7535-1121-7

ISSN : 0029-182X

\section{Référence électronique}

Brahim Akdim et Mohamed Laaouane, « Patrimoine et développement local à Fès : priorités, acteurs et échelles d'action », Norois [En ligne], 214 | 2010/1, mis en ligne le 01 juin 2012, consulté le 10 décembre 2020. URL : http://journals.openedition.org/norois/3083 ; DOI : https://doi.org/10.4000/ norois.3083 


\title{
Patrimoine et développement local à Fès : PRIORITÉS, ACTEURS ET ÉCHELLES D'ACTION
}

\author{
Brahim Akdim, Mohamed Laaouane \\ LAGEA-URAC 54 \\ (université Sidi-Mohamed-Ben-Abdellah), \\ Faculté des Lettres et des Sciences Humaines - Saïs, BP 59, FÈs, Maroc \\ akdimbrahim@yahoo.fr,laaoanemoh@yahoo.fr
}

\section{RÉSUMÉ}

L'article présente les priorités qui s'inscrivent dans la stratégie de sauvegarde et de valorisation du patrimoine urbain de la Médina de Fès. Deux principales approches différentes ont été reconnues au cours des dernières décennies. La première est mise en œuvre entre 1980 et 2000 et se caractérise par des actions ponctuelles de réhabilitation et de sauvegarde. L'article présente leurs acteurs et discute la superposition des niveaux de leur action. L'implication des acteurs locaux, régionaux et nationaux dans la sauvegarde de ce patrimoine classé sur la liste du patrimoine mondial de l'Unesco ainsi que l'intérêt porté à celle-ci par les organismes internationaux ont été observés. La seconde approche a été notée depuis 2000, car la conception et l'action dans le domaine du patrimoine et du développement local privilégient la programmation régionale et sectorielle, l'action sociale et le développement humain. Parmi les actions prioritaires actuellement mises en chantier, l'article présente deux plans régionaux, le PDRT (plan régional $d u$ développement du tourisme) et le PDRA (plan régional de développement de l'artisanat), et évoque l'intérêt porté à la réhabilitation du tissu urbain historique et la lutte contre l'habitat insalubre. Les besoins qui s'expriment dans le domaine de la réhabilitation de la Médina sont énormes en raison de ses multiples processus de dégradation. Faute de moyens financiers et d'une meilleure coordination des interventions, l'action des acteurs locaux n'arrive pas à surmonter ces défis. Vue l'ampleur de ces besoins, des interventions de niveaux plus élevés sont nécessaires.

\section{Mots CLÉS : patrimoine - développement local - Médina - Fès - Maroc}

\section{ABSTRACT \\ Cultural heritage and local development in Fez (Morocco): priorities, actors and action scales}

The article presents the priorities that appear in the local development strategies based on the valorization of the urban cultural heritage in the Medina of Fez. Two different approaches have been observed during the last decades. The first one is during the 19802000 period, when punctual and architectural actions prevailed in the Medina. The article debates the levels of responsibility and decision making in each period. The implication of the local, regional and national actors and the interest of the international organisms to the safeguard of this heritage, classified universal by the Unesco, have been observed. The second approach begun since 2000 and focuses on the regional plans and social development. Among the priorities of the action currently put in yard, the article presents the regional plans such as the PDRT (regional plan of the development of the tourism) and 
the PDRA (regional plan of development of the handicraft) and evokes the interest carried out to the rehabilitation of the urban architectural heritage and the struggle against the unsanitary habitat. The important needs expressed in this domain are in progress considering the Medina's multiple degradation processes. The action of the local actors doesn't manage to overcome these challenges due mainly, to financial problems. Interventions of more elevated levels are therefore necessary.

KEY WORDS : cultural heritage - local development - Fez - medina - Morocco

Au Maroc septentrional (fig. 1), Fès, ville historique d'envergure, est un cas de figure significatif pour l'analyse des stratégies du développement basées sur la valorisation du patrimoine. La sauvegarde et la réhabilitation de la Médina (Fès Jdid et Fès El Bali) est en chantier depuis environ trois décennies. Plusieurs actions ont été entreprises à ce propos par des acteurs locaux, nationaux et internationaux. Elles ont fait l'objet d'études sectorielles détaillées (ADER-Fès, 1996; CRI, 2004 ; MATEUH, 2002). Les impacts spatiaux, urbanistiques, fonciers et socio-économiques de ces actions ont été traités dans de nombreux travaux académiques (Ameur, 1993; Barrou, 2005; El Bouaaïchi Nadri, 2004 ; El Bouchikhi, 1990 ; Hazoui, 2006 ; Idrissi Janati, 2001 ; Lahbil-Tagemouati, 1999; Lanchet, 2006). Les dynamiques urbaines ont été appréhendées dans la littérature et la sauvegarde de la Médina a été profondément étudiée sous des angles variés (patimonial, technico-architectural, social, spirituel, environnemental, etc.). Mais l'analyse des articulations entre les échelles territoriales de l'action (locale, régionale, nationale et internationale), l'étude des effets induits par la diversité des acteurs et la faible coordination de leurs actions, restent encore peu développées. Pourtant, la concentration des interventions sur la sauvegarde de la Médina de Fès et le ralentissement du développement local des centres et zones périphériques à la fin du siècle dernier, ont engendré une flagrante segmentation territoriale à la fois à Fès et dans sa région. La Médina elle-même connaît des dysfonctionnements du système urbain engendrant des inégalités spatiales internes, qui sont traduites au niveau du paysage urbain, par les différences d'accès aux équipements collectifs et par les conditions de vie des populations. La mise en œuvre des projets d'aménagement et de sauvegarde du patrimoine urbain n’ont pas réduit ces inégalités et contrastes. Le déficit de coordination entre les intervenants et le caractère centralisateur de l'approche du développement des années quatre-vingt du siècle précédent expliquent, en partie, l'exacerbation de ces inégalités territoriales, sociales et environnementales.

Un changement de stratégies a été ensuite opéré par les différents acteurs (organismes publics, investisseurs, ONG, etc.), depuis une dizaine d'années. Le patrimoine est alors considéré comme facteur du développement local, mais pas son unique moteur. Dans cette nouvelle vision, la sauvegarde et la réhabilitation de la Médina sont toujours d'actualité, mais s'inscrivent parmi d'autres priorités de l'action. L'action sur le patrimoine et le développement local est ainsi conçue dans une perspective plus élargie. Sur le plan thématique, elle concerne des domaines variés (logement, artisanat, tourisme, industrie et développement humain par exemple) et sur le plan spatial elle intègre à la fois la Médina et les zones périphériques de Fès. Les problèmes de coordination des interventions sont mieux assimilés, car la nouvelle approche adopte l'action par programmes régionaux sectoriels concertés et contractuels entre acteurs concernés.

Sur le plan de la recherche, ce changement d'approche soulève des questions de causalités, de trajectoires et de perspectives dont les réponses éclairent de nombreux aspects des dysfonctionnements caractéristiques des stratégies antérieures. Les soubassements et aboutissants de la nouvelle approche, essentiellement axée sur le développement régional multidimensionnel, intégrent la sauvegarde et la réhabilitation du patrimoine dans des plans régionaux du développement. Les plus importants sont le Plan Régional du Développement de l'Artisanat (PDRA), le Plan Régional du Développement du Tourisme (PDRT) et l'Initiative Nationale du Développement Humain (INDH), mise en œuvre 


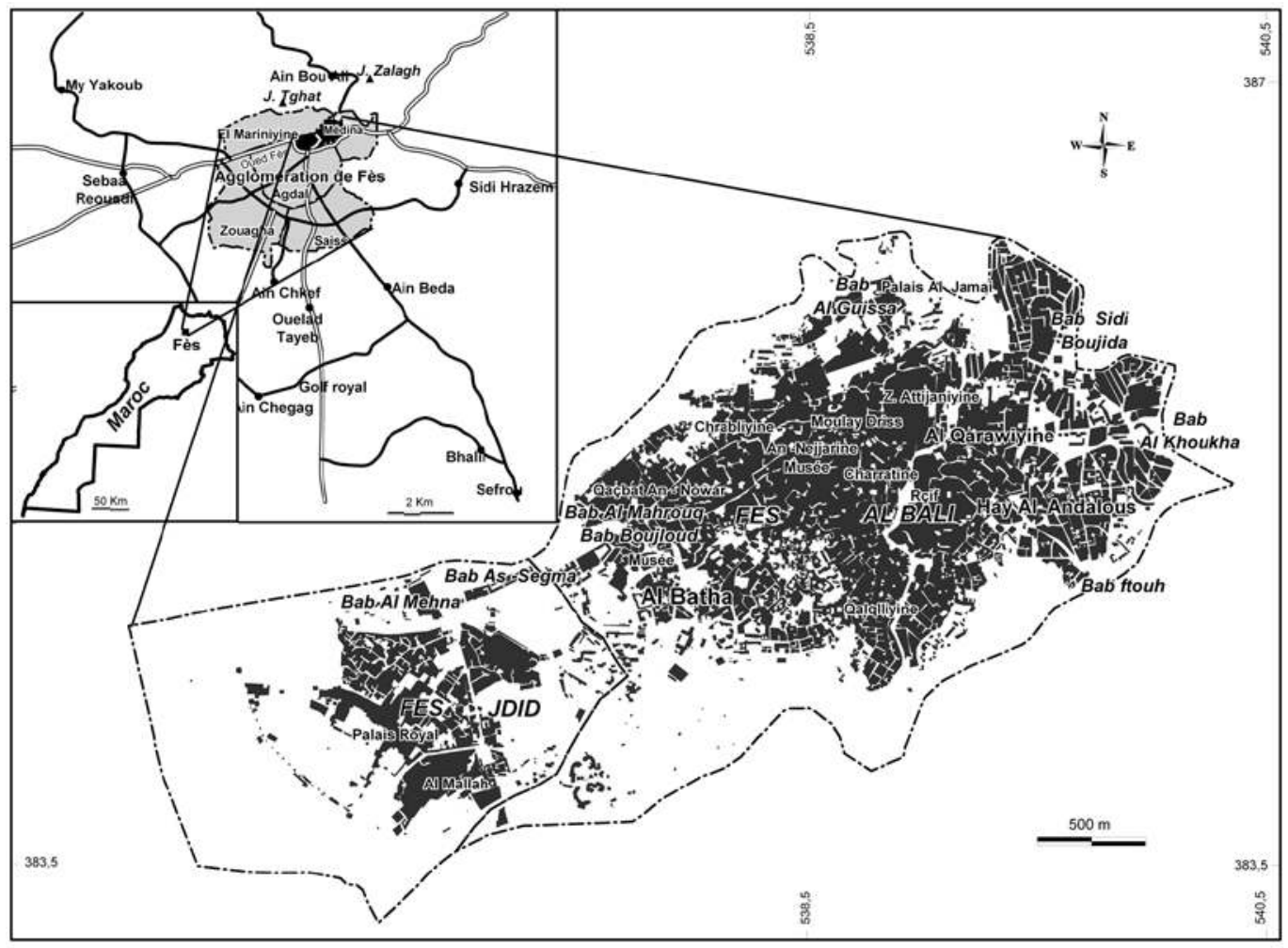

Figure 1 : Localisation de la Médina (Fès Jdid et Fès El Bali) dans l'agglomération de Fès et son contexte régional Localization of the Medina of Fez in its agglomeration and regional contexte

en Médina et dans d'autres zones sensibles de la région. La présente étude aborde le patrimoine et le développement local dans une perspective analytique et dynamique (évolutive). Nous discuterons d'abord les priorités inscrites dans la stratégie antérieurement mise en œuvre au cours des premières années de l'action et ses principaux acteurs. Dans une deuxième étape, la nouvelle approche adoptée depuis 2005 sera présentée et les défis qu’elle affronte seront analysés.

\section{Patrimoine et développement local à Fès : lancement des opérations de sauvegarde et focalisation sur la Médina}

Après un long processus de dégradation du tissu historique à Fès, l'intervention de multiples instances locales (autorités et collectivités locales), nationales (ministères, fondations, etc.) et internationales (PNUD Unesco, 1985 et Banque Mondiale) a relancé la sauvegarde et la réhabilitation de la Médina de Fès depuis la fin du précédent siècle. De nombreuses initiatives y ont été entreprises, notamment après son inscription sur la liste officielle de l'Unesco, plaçant le site sous la législation du Patrimoine mondial en 1981.

Les efforts de valorisation du patrimoine ont été multipliés par de nombreux acteurs mais les approches et la nature des actions diffèrent. La première étape (1980-1990) fût essentiellement consacrée aux études diagnostiques, avec la participation d'organismes internationaux (PNUD Unesco, 1987 et Banque Mondiale) et nationaux (autorités locales et préfectorales, départements ministériels concernés comme les ministères de l'Intérieur, de l'Habitat et de l'Urbanisme, de l'Equipement et de l'Artisanat, les organismes publics et semi publics comme l'Agence de Dédensi- 
fication et de Réhabilitation de la Médina de Fès et l'Agence Urbaine et de Sauvegarde de Fès). Le recensement des bâtisses menaçant ruine et des établissements économiques dégradés (ateliers de tissage appelés Draz, dinanderies, Foundouks, etc.) les chiffre à environ 4000 unités. Le Schéma Directeur d’Urbanisme (SDU) établi en 1980, fût le premier document réglementaire planifiant l'aménagement dans la Médina de Fès. La deuxième étape s'étendait entre 1990 et 2000 et fût l'étape du lancement des premiers grands projets de sauvegarde et de réhabilitation du patrimoine bâti. En plus des acteurs évoqués plus haut, cette étape a connu l'apparition de partenaires non gouvernementaux, qui se sont impliqués d'une façon ou d'une autre dans la sauvegarde (fondations, associations et acteurs privés). Leurs participations ne sont pas coordonnées et concernent les études, le financement, le pilotage et la réalisation des projets. L'action de sauvegarde du patrimoine urbain à Fès est influencée dans cette étape, par l'intervention d'instances internationales et nationales, qui ont tenté de relier « concepts universels, patrimoine, aménagement du territoire et développement local » en agissant sur des sites et projets ponctuels. Les échelles de conception et d'action sont donc très variées, bien que la plus visible reste locale, à travers les travaux menés sur des bâtisses et monuments ciblés. Cette action d'envergure a mobilisé des experts délégués d'organisations internationales (Unesco, PNUD) et des experts européens et marocains (Groupe Huit, Iskane) impliqués dans des études de faisabilité du projet. La Banque Mondiale, qui, pour la première fois s’implique dans le financement de projets de ce type, expérimente dans la Médina de Fès la stratégie qui mise sur le développement social pour impliquer la population dans le processus de réhabilitation et de sauvegarde de la Médina.

Les instances du niveau national, représentées par les différents ministères concernés (Habitat, Aménagement du Territoire, Environnement, Intérieur, etc.), sont intervenues d'une façon ou d'une autre dans la sauvegarde. Celle-ci a d'abord été soutenue par des mesures réglementaires et institutionnelles (établissement du Schéma Directeur d'Urbanisme et création de l'Agence pour la Dédensification et la Réhabilitation de la Médina de Fès) puis par l'implication dans le financement et la réalisation des projets. La création de l'Agence de Dédensification et Réhabilitation de la Médina de Fès est une mesure institutionnelle de grande importance vu son statut autonome et ses prérogatives en matière de sauvegarde et de réhabilitation du patrimoine. Elle a investi, au cours des deux dernières décennies, dans la sauvegarde de la Médina et la lutte contre l'habitat insalubre. En plus des études, les actions de cet organisme sont aussi importantes dans le domaine de l'étayement des bâtisses les plus sensibles (fig. 2 - planche I), l'encouragement des propriétaires à réparer leur maison et l'encadrement technique notamment. Avec la commune urbaine et les autorités compétentes, de nombreux partenariats ont été élaborés dans le cadre de cette stratégie opérationnelle qui commence à donner ses résultats positifs au niveau de la maîtrise des risques liés à l'insalubrité de l'habitat en milieu urbain. L'effet local de ces mesures est rapidement apparu avec la réalisation de nombreux projets pilotes (étayement et actions d'urgence sur la structure des immeubles privés, restauration du Fondouk Nejjarine, de la Medersa Bouaanania, conception de projets structurants comme celui du transfert des activités artisanales polluantes à l'extérieur de la Médina par exemple). Le programme de restauration, de réhabilitation et de sauvegarde des sites et monuments de la ville couvre d'autres actions d'équipement et d'aménagement (percées, portes et pénétrantes, dallage des ruelles, aménagement des places Rcif et Boujloud, aménagement de Oued El Jawahir, équipement des circuits touristiques de la Médina, aménagement du secteur de Ain Azliten et de l'entrée de Rcif, etc.). Ces actions ont été lancées dans un cadre de partenariats impliquant de nombreux acteurs locaux, nationaux et internationaux.

Le tableau 1 donne, à titre indicatif, les principaux intervenants dans la sauvegarde et le développement local, à travers les grands chantiers de Fès. On note la diversité de ces acteurs et la nécessité de développer les cadres de coordination appropriés pour éviter les confusions parfois observées, des limites entre les domaines de compétences de certains acteurs institutionnels.

Au cours de cette étape, les interventions ont été ponctuelles et localisées et touchent en premier lieu les constructions menaçant ruine ainsi que certains monuments et sites de grande valeur patrimoniale (Foundouks, remparts et places publiques). Le projet de transfert des activités 


\begin{tabular}{|c|c|c|c|c|}
\hline Plan & Local & Régional & National & International \\
\hline $\begin{array}{l}\text { Sauvegarde de la } \\
\text { Médina }\end{array}$ & $\begin{array}{l}\text { - collectivités } \\
\text { locales, } \\
\text { - autorités locales, } \\
\text { - ADER Fès, } \\
\text { - AUSF, } \\
\text { - RADEEF, } \\
\text { - CRT, } \\
\text { - université, } \\
\text { - ONG, } \\
\text { - privé, etc. }\end{array}$ & $\begin{array}{l}\text { - préfecture } \\
\text { (Wilaya) } \\
\text { - région Fès } \\
\text { - Boulemane } \\
\text { - services externes } \\
\text { des ministères, } \\
\text { - Al Omrane }\end{array}$ & $\begin{array}{l}\text { - ministères (Habi- } \\
\text { tat, Aménagement } \\
\text { du Territoire, Envi- } \\
\text { ronnement Équipe- } \\
\text { ment, etc.) } \\
\text { - fonds et fonda- } \\
\text { tions }\end{array}$ & $\begin{array}{l}\text { - bailleurs de fonds } \\
\text { - PNUD } \\
\text { - banque Mondiale } \\
\text { - Unesco } \\
\text { - ONG } \\
\text { - programmes de } \\
\text { coopération etc. }\end{array}$ \\
\hline $\begin{array}{l}\text { Plan de développe- } \\
\text { ment régional tou- } \\
\text { ristique (PDRT) }\end{array}$ & $\begin{array}{l}\text { - collectivités } \\
\text { locales } \\
\text { - autorités locales } \\
\text { - ADER Fès } \\
\text { - AUSF } \\
\text { - RADEEF } \\
\text { - CRT } \\
\text { - université } \\
\text { - ONG, Privé, etc. }\end{array}$ & $\begin{array}{l}\text { - préfecture } \\
\text { (Wilaya) } \\
\text { - région Fès Bou- } \\
\text { lemane } \\
\text { - services externes } \\
\text { des ministères, } \\
\text { - Al Omrane }\end{array}$ & $\begin{array}{l}\text { - ministères } \\
\text { - fonds et Fon- } \\
\text { dations }\end{array}$ & $\begin{array}{l}\text { - opérateurs inter- } \\
\text { nationaux }\end{array}$ \\
\hline $\begin{array}{l}\text { Plan de } \\
\text { Développement } \\
\text { Régional de l'Arti- } \\
\text { sanat (PDRA) }\end{array}$ & $\begin{array}{l}\text { - collectivités } \\
\text { locales } \\
\text { - autorités locales } \\
\text { - ADER Fès } \\
\text { - AUSF } \\
\text { - RADEEF } \\
\text { - CRT } \\
\text { - université } \\
\text { - ONG, Privé, etc. }\end{array}$ & $\begin{array}{l}\text { - préfecture } \\
\text { (Wilaya) } \\
\text { - région Fès Bou- } \\
\text { lemane } \\
\text { - services externes } \\
\text { des ministères, } \\
\text { - Al Omrane }\end{array}$ & $\begin{array}{l}\text { - ministères } \\
\text { - fonds et fon- } \\
\text { dations }\end{array}$ & $\begin{array}{l}\text { - programmes de } \\
\text { coopération }\end{array}$ \\
\hline $\begin{array}{l}\text { Initiative Locale } \\
\text { du Développement } \\
\text { Humain } \\
\text { (ILDH) }\end{array}$ & $\begin{array}{l}\text { - collectivités } \\
\text { locales } \\
\text { - autorités locales } \\
\text { - ADER Fès } \\
\text { - AUSF } \\
\text { - RADEEF } \\
\text { - CRT } \\
\text { - université } \\
\text { - ONG, Privé, etc. }\end{array}$ & $\begin{array}{l}\text { - préfecture } \\
\text { (Wilaya) } \\
\text { - région Fès Bou- } \\
\text { lemane } \\
\text { - services externes } \\
\text { des ministères, } \\
\text { - Al Omrane }\end{array}$ & $\begin{array}{l}\text { - ministères, } \\
\text { - fonds et fon- } \\
\text { dations }\end{array}$ & $\begin{array}{l}\text { - programmes de } \\
\text { coopération }\end{array}$ \\
\hline
\end{tabular}

(BM : Banque mondiale; ADER Fès : Agence de la dédensification et de la réhabilitation de la Médina de Fès; RADEEF : Régie autonome de distribution de l'eau et électricité; AUSF : Agence urbaine et de sauvegarde de Fès; ILDH : Initiative locale du développement humain; CRT : Centre régional du tourisme).

Tableau 1 : Acteurs et échelles d'intervention dans la sauvegarde et le développement local à Fès Actors and intervention scales in the cultural heritage safegard and local development within Fez

artisanales polluantes au quartier Aïn Nokbi, situé à l'extérieur de la Médina a été également lancé. Les travaux d'équipement du site ont été réalisés, mais le transfert n'a pas encore eu lieu, à cause de la réticence des artisans concernés. Ceux-ci craignent la perte des avantages économiques du site (proximité du marché notamment).

L'évaluation des acquis en matière de sauvegarde et de réhabilitation du patrimoine dans la Médina de Fès avant 2000 montre une divergence d'avis. Certains auteurs critiquent l'approche mise en œuvre, sous-tendant un savoir « expert » et «l'idéologie patrimoniale institutionnelle » voulant fabriquer en Médina l'espace « modèle » conçu d'en haut (Lanchet, 2006). D’autres 
critiquent l'approche technique et architecturale qui a été mise en œuvre dans cette étape et préviligient l'approche sociale (Navez - Bouchanine, 2002 et 2005). D'autres encore mettent l'accent sur la nécessité d'intégrer les perceptions et les pratiques d'acteurs « ordinaires » et les réalités sociales dans tout projet de sauvegarde, pour une meilleure maîtrise de l'ouvrage social (El Bouaaïchi Nadri, 2004). Ce sont des actions illustrant le cumul scientifique généré par l'expérience menée à Fès et l'importance des réflexions engagées à ce propos sur le plan académique. Il est vrai que les résultats matériels et socio-économiques acquis, comparés aux attentes, peuvent être discutés, mais, vu l'ampleur des problèmes urbanistiques et socio-économiques de la Médina, leurs solutions n'auront d'effets que par des actions structurelles, à des échelles spatiales variées et sur des périodes suffisamment longues pour traiter les dysfonctionnements territoriaux à Fès dans leur contexte le plus élargi.

\section{Changement de l'approche : quand l'approche régionale et le développement humain priment}

Conformément aux orientations nationales ciblant le renforcement de la régionalisation, les processus de sauvegarde et de réhabilitation du patrimoine dans la Médina de Fès se sont inscrits, depuis le début des années 2000, dans une nouvelle stratégie, qui privilégie l'action régionale et le développement humain. C'est la troisième étape de l'évolution des stratégies du patrimoine et du développement de Fès.

L'approche régionale se base sur des secteurs porteurs du développement et des créneaux d'attractivité territoriale comme le tourisme et l'artisanat. L'approche sociale quant à elle, vise la lutte contre l'insalubrité et l'exclusion sociale. Les deux approches convergent en matière de soutien aux initiatives de sauvegarde et de réhabilitation du patrimoine culturel (architectural et économique) dans la Médina et élargissent le domaine de leur action à des échelles plus élargies. À la différence de l'approche précédente (mise en œuvre avant 2000), essentiellement focalisée sur des sites sélectionnés dans la Médina, la nouvelle approche espère promouvoir les dynamiques internes du développement, en facilitant les interférences positives de systèmes territoriaux multiples, de paramètres variés, à des échelles de prise de décision distinctes, au sein de la grande région administrative. La Médina de Fès, qui continue son action de mise à niveau urbaine et architecturale, doit en plus s'inscrire dans des chantiers du développement régional, lancés et mis en œuvre dans un cadre contractuel, qui définit les échéances et les responsabilités des intervenants dans chaque projet. La métropole profitera de ces chantiers sur le plan économique et social. Nous présentons, ici, deux chantiers considérés comme significatifs pour le patrimoine et le développement local à Fès : le tourisme et l'artisanat.

\section{L'approche régionale du développement du tourisme : mise en œuvre du Plan de Développement Régional Touristique (PDRT)}

Suivant cette approche, un Plan de Développement Régional Touristique (PDRT), qui est d'ailleurs le premier à être conçu à l'échelle nationale, a été mis en place. Il s'agit d'un programme diversifié élaboré selon une vision prospective et un projet clair de développement intégrant toutes les composantes de l'activité comme l'accueil, l'hébergement, l'animation, l'intégration d'autres formes du tourisme (comme le tourisme culturel) et la préparation des structures de communication et de transport. De nombreux projets sont programmés comme l'aménagement de la zone touristique de l'Oued Fès et la création de la zone touristique de l'Oued Ouislane (fig. 1). En Médina, le PDRT vient consolider les actions de réhabilitation et de restauration de nombreux monuments historiques, qui peuvent être réutilisés à d'autres fins, comme l'animation culturelle. Une considérable dynamique d'investissement en Médina est en cours dans le cadre du développement de nouvelles formes d'accueil touristique. C'est le cas des maisons d'hôtes souvent fondées dans des 
riads restaurés pour un accueil de luxe. Le rayonnement historique, religieux et culturel de Fès a été pris en considération dans ce cadre. Le Conseil Régional du Tourisme (CRT), chargé de la réalisation et du suivi du PDRT, apporte son soutien à la promotion de cette activité à des fins touristiques. La Zaouia Sidi Ahmed Tijani, connue dans de nombreux pays africains et la prestigieuse mosquée Karawiyine sont, entre autres, des sites ayant un grand rayonnement spirituel et participent à la réussite de cette stratégie. Dans la même optique, plusieurs circuits touristiques urbains ont été conçus. L'accueil touristique s'est ainsi développé considérablement au cours de cette décennie et se rapproche actuellement d'une capacité d'environ 10000 lits. Le lancement des projets de Ouislane et de Oued Fès renforcera considérablement cette capacité.

Comme cela a été évoqué dans le Schéma d'orientation et de structuration de la zone périphérique de la ville de Fès, en améliorant son offre traditionnelle, la ville a également intérêt à composer avec son environnement proche et régional, pour diversifier cette offre et permettre des séjours touristiques plus longs et davantage orientés vers l'environnement naturel régional (la montagne Jbel Tghat est à $2 \mathrm{~km}$ de la ville, les sources de Aïn Chkef et Ras El Ma sont à environ $7 \mathrm{~km}$; les thermes de Moulay Yacoub sont à $18 \mathrm{~km}$ et celles de Sidi Hrazem à $15 \mathrm{~km}$; le terrain du Golf de Aïn Cheggag est à $21 \mathrm{~km}$ ) (fig. 1). Le PDRT intègre donc l'exploitation des atouts de ce contexte régional pour développer le tourisme de séjour à Fès. La vision antérieure du développement touristique en focalisant sur la seule Médina est donc dépassée.

\section{Le Plan de développement régional de l'artisanat (PDRA), 2007-2011}

C'est un programme de développement stratégique proposé par le ministère de l'Artisanat et élaboré en concertation avec de nombreux acteurs intéressés, dont notamment les collectivités locales, les chambres professionnelles et les associations d'artisans. Considérant le potentiel de la ville de Fès en matière d'artisanat, le programme pour la mise à niveau du secteur de l'artisanat (PDRA) a été élaboré pour renforcer le rôle de la ville comme centre des arts traditionnels et en faire une façade de l'artisanat national. Le projet a été signé et mis en œuvre depuis septembre 2007 par les acteurs suivants : Wilaya, ministère du Tourisme, de l'Artisanat et de l'Économie Sociale, Région Fès Boulemane, commune de Fès, chambre de l'Artisanat à Fès et le Conseil provincial de Fès. C'est un exemple de partenariat impliquant des acteurs multiples à des échelles variées (locale, régionale et nationale).

Le programme est composé de quatre principaux axes qui s'articulent autour de plusieurs actions.

Le premier axe vise l'appui à la production de l'artisan individuel. Il met l'accent sur des actions de motivation des producteurs pour le développement des produits de qualité. Il s'agit des actions suivantes :

- la mise en place d'un programme de formation de designers. Ceci permettra d'avoir sur le marché des produits au label «made in Fès » en mesure de soutenir la compétitivité de ce produit sur le marché national et international;

- la réalisation d'études techniques pour la conception et l'amélioration du produit conformément aux normes du développement durable;

- la valorisation de zones d'activité dédiées à l'artisanat, comme le quartier des dinandiers de Ain Nokbi, les foundouks d'entrepreneurs d'artisanat, le quartier artisanal de Ben Jellik, etc.;

- la généralisation de l'usage des fours à gaz (énergie peu polluante) pour l'artisanat;

- la maîtrise des réseaux de commercialisation des matières premières nécessaires pour l'artisanat;

- la réhabilitation et sauvegarde des métiers d'artisanat menacés de disparition;

- la création d'un système de contrôle de la qualité « labellisation » du produit.

Le deuxième axe cible l'amélioration de la commercialisation des produits des artisans individuels par les actions suivantes : 
- multiplication des sites de vente et valorisation des places et sites de commercialisation (Place Lalla Yaddouna, Place Bab El Makina, Foundouks Chemaiine, Sbitriyine, Staouniyine et El Berka);

- réaménagement du complexe artisanal de Fès;

- création de zones de commercialisation des produits d'artisanat dans les nouvelles zones touristiques qui seront créees à Oued Ouislane et Oued Fès ;

- appui à la promotion (publicité) du produit d'artisanat ;

- soutien à l'exportation des produits d'artisanat commercialisés.

Le troisième axe, ciblant le développement et la restructuration de l'entreprise dans le domaine de l'artisanat, projette les actions suivantes :

- appui aux PME existantes;

- création de nouvelles PME d'artisanat;

- création de zones d'accueil de ces PME.

Le quatrième axe, intitulé « mesures horizontales », cible :

- la promotion des ventes de produits d'artisanat aux touristes, la commercialisation à l'échelle nationale, l'exportation des produits d'artisanat, le contrôle de la qualité des produits, l'encadrement des opérations de commercialisation, le soutien à l'artisanat rural ;

- la formation des artisans et l'application des orientations du Plan Directeur de la Formation Professionnelle aux métiers de l'artisanat, la réhabilitation et la mise à niveau du Centre de formation par la pratique à Aïn Kadous, la réouverture de l'Institut des arts traditionnels de Fès, la mise aux normes du Centre de formation professionnelle de Sefrou, la formation continue au profit des artisans, ainsi que l'élaboration de nouveaux modes de financement, la couverture sociale, la création d'un registre de l'artisan, la création d'un centre de comptabilité, l'encouragement de l'organisation structurée des artisans, le développement des partenariats et jumelages. Un montage financier du programme a d'ores et déjà été élaboré.

À terme, ce programme sera en mesure de générer des impacts positifs sur le plan économique et social, et contribuer ainsi au développement local dans la Médina et son contexte régional. Mais son lancement récent ne permet pas à présent d'entamer l'évaluation objective de ses résultats.

\section{L'Initiative Nationale du Développement Humain (INDH) : nouvelle approche de mise à niveau urbaine et d'intégration sociale}

Depuis son lancement en 2005, l'Initiative Nationale du Développement Humain représente un chantier prioritaire de lutte contre l'insalubrité et l'exclusion sociale. Ces grands défis sont observés, notamment en Médina et dans les quartiers périphériques sous équipés, de la ville de Fès. D'importants moyens ont été mis en œuvre pour atteindre les objectifs du développement humain à Fès. Un programme local (appelé Initiative Locale du Développement Humain ou ILDH) a été adopté et son application montre des avancées dans la mise à niveau urbaine sur le plan de la lutte contre l'habitat insalubre, l'offre en logement, le développement des espaces verts, les équipements collectifs, etc. Les actions évoquées dans le cadre de la sauvegarde de la Médina ont grandement contribué à cette actualisation urbaine, bien que le tissu historique reste fragile et très vulnérable face aux mécanismes de dégradation (tableau 2).

D'importantes interventions ont été ensuite entreprises dans le cadre de l'ILDH par les pouvoirs publics, les ONG et les populations, pour lutter contre l'habitat insalubre dans la Médina, créer des activités génératrices de revenus et lutter contre la pauvreté et l'exclusion sociale. Plusieurs projets sont financés et réalisés annuellement. À titre d'exemple, les actions programmées en Médina dans le cadre du programme transversal de l'ILDH pour la seule année 2007 illustrent la diversité des projets, l'implication d'acteurs multiples dans ces projets et l'importance des budgets investis dans la Médina (tableau 3). 


\begin{tabular}{|l|c|c|c|c|}
\hline & Nombre de bâtisses & Nombre d'opérations & \multicolumn{2}{|c|}{ Nombre d'opérations achevées } \\
\cline { 3 - 5 } & menaçant ruine & lancées & Bâtisses & Ménages \\
\hline Fès El Bali & 874 & 669 & 566 & 2094 \\
\hline Fès Jdid & 268 & 148 & 128 & 474 \\
\hline Total & 1142 & 817 & 694 & 2568 \\
\hline
\end{tabular}

Tableau 2 : Réalisations en matière de lutte contre l'insalubrité et l'habitat mençant ruine dans la Médina de Fès en 2005 (Sources : ADER-Fès dans El Bouchikhi et al., 2005)

Achieved results of fight against insalubrity and degraded habitat in the Fez Medina in 2005

\begin{tabular}{|l|c|c|c|c|}
\hline \multicolumn{1}{|c|}{ Programme transversal } \\
\hline \multicolumn{1}{|c|}{ Nature du Projet } & $\begin{array}{c}\text { Porteur du } \\
\text { projet }\end{array}$ & $\begin{array}{c}\text { Coût global en } \\
\text { dirhams }\end{array}$ & $\begin{array}{c}\text { Financement } \\
\text { du partenaire } \\
\text { public en } \\
\text { dirhams }\end{array}$ & $\begin{array}{c}\text { Financement } \\
\text { de l'ILDH en } \\
\text { dirhams }\end{array}$ \\
\hline Réhabilitation des Fondouks & ILDH & 1300000 & & 1300000 \\
\hline $\begin{array}{l}\text { Mise à niveau du réseau d'assainisse- } \\
\text { ment }\end{array}$ & ILDH & 2400000 & $\begin{array}{c}\text { Commune } \\
\text { urbaine et } \\
\text { RADEEF }\end{array}$ & 720000 \\
\hline $\begin{array}{l}\text { Réhabilitation des ateliers de maro- } \\
\text { quinerie et cuir (Aïn Azliten et Sidi } \\
\text { Moussa) }\end{array}$ & ILDH & 2000000 & & 2000000 \\
\hline $\begin{array}{l}\text { Création d'une bibliothèque multi- } \\
\text { média - maison de la culture dans la } \\
\text { Medersa Med Ben Youssef }\end{array}$ & ILDH & 8000000 & & 1000000 \\
\hline $\begin{array}{l}\text { Aménagement d'un espace de ven- } \\
\text { deurs ambulants }\end{array}$ & ILDH & 1000000 & & \\
\hline $\begin{array}{l}\text { Appui à l'association « Assadaka » pour } \\
\text { le soutien aux plus démunis }\end{array}$ & $\begin{array}{l}\text { Entraide } \\
\text { nationale }\end{array}$ & 30000 & 30000 & \\
\hline $\begin{array}{l}\text { Équipement de la maison du citoyen } \\
\text { Batha pour l'accueil des populations en } \\
\text { situation critique }\end{array}$ & $\begin{array}{l}\text { Entraide } \\
\text { nationale }\end{array}$ & 200000 & 200000 & \\
\hline
\end{tabular}

Tableau 3 : Financement des actions de l'Initiative Locale du Développement Humain (ILDH) pour la mise à niveau urbaine et la lutte contre la pauvreté et l'exclusion sociale dans la Médina de Fès en 2007 (100 dirhams 9 euros). Sources : INDH, Fès, 2007

Funding of the Local Initiative for the Human Development (ILDH) projects for the urban upgrading and the struggle against poverty and the social exclusion in the Medina of Fez in 2007 (100 dirhams $~$ 9 euros)

Ces actions s'inscrivent dans le cadre du programme transversal de l'ILDH, qui n'est qu'une composante parmi ses programmes d'intervention. Le tableau 4, donné à titre d'exemple, résume les principales interventions sectorielles de l'ILDH à Fès en 2007.

Les exemples cités dans le tableau 4 illustrent la nouvelle approche mise en œuvre par l'INDH/ ILDH pour la mise à niveau urbaine, la lutte contre l'habitat insalubre et contre la pauvreté. L'approche adoptée par l'INDH privilégie le partenariat entre acteurs variés et participation de la population concernée à la conception, la réalisation et le suivi de chaque projet. La priorité est donnée aux projets de proximité comme l'instauration des équipements collectifs et infrastructures de base dans le quartier, la création d'activités génératrices de revenus et la réhabilitation du patrimoine culturel. 


\begin{tabular}{|c|c|c|}
\hline \multicolumn{3}{|c|}{ Projets sectoriels programmés en 2007} \\
\hline Axe d'intervention & Typologie de projets & Nombre de projets \\
\hline \multirow{5}{*}{$\begin{array}{l}\text { Amélioration de l'accès aux équipe- } \\
\text { ments et services sociaux de base }\end{array}$} & Dallage des voies et pavage des ruelles & 05 \\
\hline & $\begin{array}{l}\text { Réhabilitation des réseaux d'assainisse- } \\
\text { ment }\end{array}$ & 06 \\
\hline & Évacuation des ruines & 05 \\
\hline & $\begin{array}{l}\text { Réhabilitation et création des espaces } \\
\text { verts }\end{array}$ & 04 \\
\hline & $\begin{array}{l}\text { Confortement d'une bâtisse menaçant } \\
\text { ruine }\end{array}$ & 01 \\
\hline \multirow{4}{*}{ Animation culturelle, sociale et sportive } & $\begin{array}{l}\text { Construction et équipement des mai- } \\
\text { sons du citoyen et du quartier }\end{array}$ & 05 \\
\hline & $\begin{array}{l}\text { Construction et équipement des mai- } \\
\text { sons des jeunes, des clubs d'enfants et } \\
\text { foyers féminins }\end{array}$ & 06 \\
\hline & $\begin{array}{l}\text { Constructions et équipement des biblio- } \\
\text { thèques }\end{array}$ & 02 \\
\hline & $\begin{array}{l}\text { Construction et réhabilitation des salles } \\
\text { couvertes omnisport et terrains de sport }\end{array}$ & 07 \\
\hline \multirow{2}{*}{$\begin{array}{l}\text { Appui aux activités génératrices de } \\
\text { revenus }\end{array}$} & $\begin{array}{l}\text { Aménagement et équipement d'une aire } \\
\text { de vente }\end{array}$ & 01 \\
\hline & Projet « tourisme chez l'habitant » & 01 \\
\hline \multicolumn{2}{|r|}{ Total } & 43 \\
\hline
\end{tabular}

Tableau 4 : Interventions sectorielles de l'Initiative Locale du Développement Humain dans la Médina de Fès en 2007 (Source : INDH, Fès, 2007)

2007

Projects realized on behaf of the Local Initiative of Human Development at the Medina of Fez, in

\section{Émergence des acteurs locaux : rôles, perceptions et contraintes}

La nouvelle approche du développement régional sectoriel et de l'INDH favorise l'émergence des nouveaux acteurs locaux du développement territorial. Ceux-ci sont considérés comme partenaires dans toutes les étapes des projets (conception, réalisation et suivi). Les artisans, les commerçants et d'autres acteurs locaux sont appelés à soumettre des projets pour bénéficier des financements réservés au développement humain, à l'équipement des quartiers, à la sauvegarde et à la réhabilitation du patrimoine. Cette stratégie partenariale en faveur des acteurs « ordinaires » offre, par exemple, aux propriétaires des habitations menaçant ruine dans la Médina la possibilité de bénéficier d'un soutien financier pouvant atteindre $50 \%$ du coût global de la restauration de leurs demeures, si celui-ci est inférieur à 120000 dirhams. La mise en œuvre de cette stratégie est encore récente pour évaluer ses résultats, mais son impact potentiel peut être important si la population concernée est sensibilisée pour accroître le nombre de bénéficiaires. À présent, la promotion de cette stratégie reste insuffisante. Les résultats de nos enquêtes illustrent des écarts entre le discours des acteurs institutionnels (ADER Fès par exemple) et les perceptions de certains propriétaires qui espèrent bénéficier d'une prise en charge totale des frais de la restauration de leurs demeures. Les fonds consacrés au financement de ces programmes proviennent d'un montage financier basé sur la contribution du Budget général de l'État et la participation des bayeurs de fonds, mais plusieurs interviewés mettent l'accent sur le financement de l'Unesco, qui pour eux, « serait suffisant pour financer la totalité des frais de la sauvegarde et doit être distribué comme 
dons aux populations ». Des confusions d'avis apparaissent au sujet des origines des fonds mis à la disposition de ces programmes. Elles persistent même au niveau de la connaissance des acteurs institutionnels concernés. Plusieurs personnes interrogées ignorent la différence entre l'ADER Fès et l'AUSF (Agence Urbaine et de Sauvegarde de Fès) ainsi que leurs statuts et compétences. Un déficit de communication est donc observé à ce propos, ce qui entrave l'adhésion de nombreux intéressés, aux stratégies mises en œuvre.

Le finacement de l'INDH quant à elle, provient du budget général de l'État (compte spécial de soutien à l'INDH), des collectivités locales et de la coopération internationale. Les critères de sélection des projets financés sont clairement définis et publiquement diffusés et mettent l'accent sur la participation des ONG. Ainsi, les organisations non gouvernementales sont appelées à s'impliquer dans ces programmes et les populations concernées sont encouragées à s'organiser en associations ou coopératives de quartiers pour bénéficier des financements de leurs projets dans le cadre de l'INDH. Une nouvelle dynamique d'organisation sociale est donc lancée. Ses enjeux sont stratégiques car:

- elle peut changer le mode de gestion locale avec la participation de nouveaux acteurs associatifs ;

- elle affecte positivement la vie des populations en zones sensibles;

- elle offre aux ONG, des opportunités de concevoir, financer et réaliser leurs projets.

L'évaluation des résultats obtenus par ces approches novatrices est précoce. Mais on peut reconnaitre les contraintes de leur mise en œuvre, vu la jeunesse de la majorité des associations impliquées et leur faible expérience en matière de montage et de gestion des projets. Donc, d'énormes efforts de sensibilisation et de formation sont nécessaires. En effet, les acteurs locaux doivent assimiler les mutations en cours et comprendre leurs missions et responsabilités. Ils doivent aussi connaître et saisir les opportunités de financement de leurs projets dans ce nouveau contexte.

Par ailleurs, au cours des dernières années, la Médina de Fès connaît l'émergence de nouveaux acteurs privés du développement local, comme par exemple les promoteurs et investisseurs des maisons d'hôtes. Cette nouvelle forme d'accueil touristique est aussi un nouveau mode de réhabilitation du patrimoine et du développement touristique, car la majorité des bâtisses transformées en maisons d'hôtes ont souvent une valeur historique et patrimoniale. Certaines étaient des ruines et sont réhabilitées dans ce cadre. Le phénomène prend davantage d'ampleur avec la participation d'investisseurs étrangers. Ce nouveau mode d'hébergement se développe et commence à attirer un type de clientèle aisée et intéressée au produit culturel. Les maisons d'hôtes deviennent progressivement un service d'accueil compétitif et leur nombre s'accroît considérablement; elles passent de vingt-deux, en 2004 à plus de quatre-vingt en 2009. La diversité et l'apparition de ces nouveaux acteurs du développement économique et social sont liées aux dynamiques du développement local de Fès.

\section{Conclusion}

Suite aux précédentes réflexions, on voit que la sauvegarde et la réhabilitation du patrimoine ainsi que le développement local de Fès s'inscrivent dans une perspective dynamique aux effets territoriaux et socio-économiques considérables. L'approche qui a été menée au cours des trois dernières décennies, a connu des changements de priorités, d'échelles de l'action et d'acteurs. La sauvegarde et le développement local de Fès ont intéressé plusieurs instances locales, régionales, nationales et même internationales. À travers l'implication notamment de l'Unesco, de la Banque Mondiale et du PNUD, «l'international » est donc omniprésent dans le développement local à Fès. Mais dans le secteur de l'habitat menaçant ruine, l'intervention est essentiellement nationale, voire locale dans le cadre des activités de proximité menées par l'ADER et l'ILDH. L'ampleur des besoins montre que les moyens disponibles au niveau local seraient, en tout cas, insuffisants pour affronter les défis. Une plus intense implication des acteurs à des échelles plus élevées s’impose, notamment au niveau du soutien financier et du financement des projets. 
Mais au-delà des moyens, pouvant être disponibles dans le cadre de partenariats et de l'INDH, le besoin le plus pressant est à notre avis la sensibilisation, le renforcement des aptitudes des acteurs locaux, notamment associatifs, en matière de conception et de gestion des projets, leur sensibilisation aux opportunités offertes en matière de financement de projets et donc leur encouragement à faire des propositions d'idées novatrices pour sauvegarder le patrimoine, développer la cité et améliorer, en partenariat avec d'autres acteurs concernés, les conditions de vie des populations.

Il est vrai qu'en comparant les dynamiques de sauvegarde et de développement actuellement en cours à Fès avec celles des années quatre-vingt, on peut conclure que la relance de la ville est bien enclenchée et que la Médina retrouvera progressivement son essor. Mais l'engagement des élites et des entrepreneurs par le biais de projets novateurs est plus que sollicité. Le dynamisme des contextes locaux, régionaux, nationaux et internationaux impose aux acteurs du développement local de Fès, de renouveler en permanence leurs approches. Les grands chantiers actuellement ouverts engendrent des dynamiques positives et un développement local illustré par de nombreux indicateurs (emploi, qualité des paysages, infrastructures, prix du foncier, etc.). L'agglomération de Fès en général et la Médina en particulier, attirent des investissements nationaux et étrangers, mais les inégalités internes du développement local restent notables. Des plans sectoriels du développement, ciblant les zones encore sensibles et l'implication progressive des populations concernées, permettront une meilleure visibilité du futur.

L'imbrication des échelles d'intervention et des types d'acteurs et l'apparition de nouveaux acteurs notamment locaux, fait de Fès le terrain d'enjeux sociaux, économiques, culturels et environnementaux complexes. Les interactions du niveau local, régional, national et international se renforcent, faisant de cette ville rayonnante à travers l'histoire, une aire ouverte et dynamique mais encore vulnérable et très sensible.

\section{Bibliographie}

ADER-Fès (Agence pour la Dédensification et la Réhabilitation de la Medina de Fès), 1996. Sauvegarde de la Médina de Fès (études préliminaires), Editions Scandiaconsult, rapport final, 180 p.

Ameur (M.), 1993. Fès ou l'obsession du foncier. Fascicule de Recherche n² 25, URBAMA, Université de Tours, $428 \mathrm{p}$.

Barrou (B.), 2005. Fès, de la gestion urbaine normative à la gouvernance, Éditions INAU, 400 p.

Centre Régional d’Investissement (CRI), 2004. Fès-Boulemane : une région en action, Livret guide du CRI, Fès, 15 p.

El BouaAïchi NAdri A., 2004. Les périphéries urbaines de Fès en action de mise à niveau : la recomposition de la ville, les pratiques et les alternatives d'aménagement, Thèse de doctorat d'État en géographie, Faculté des Lettres Saïs, Fès, 709 p.

El Bouchiкhi M., 1990. La rénovation de l'habitat dans la Médina de Fès. Thèse de Doctorat, Université de Bordeaux III, $304 \mathrm{p}$.

El Bouchiкhi M., Taleb A., Акdiм B., 2005. La gestion des risques urbains dans la Médina de Fès. p. 194-208 «Aménagement du Territoire et gestion des risques environnementaux », dans Акдім B. et LaAouane M. (dir.), Publications de l'université Sidi-Mohamed-Ben-Abdellah, Fès, 640 p.

Hazour M., 2006. La ville marocaine entre la planification et l'anarchie. Publication de la FLS, Fès, Série colloques $n^{\circ} 5,489 \mathrm{p}$.

IDRISSI JanATI, M., 2001. Les jeunes des quartiers populaires de Fès : représentations sociales et territorialités urbaines. Thèse de doctorat, Université François-Rabelais, Tours, URBAMA, 505 p.

INDH, 2007. http://www.indh-fes.ma/presse/107-restauration-renovation-projets-structuraux.

Lahbil-Tagemouati N., 1999. «Emploi et projet de développement intégé - le cas de la sauvegarde de la Médina de Fès ", Revue de Droit et d'Économie, FSJE, Fès, nº 17, p. 111-123.

LANCHET W., 2006. La ville entre concepteurs et usagers : problématique de la sauvegarde appliquée au cas de la médina de Fès (Maroc), Thèse de Doctorat, Université François-Rabelais Tours, URBAMA », 346 p. 
MATEUH (ministère de l'Aménagement du Territoire, de l'Environnement, de l'Urbanisme et de l'Habitat), 2002. Plan Local de l'habitat et du développement urbain de Fès, Rapport final, Rabat, 96 p.

Navez-Bouchanine (F.), 2002. - La fragmentation en question, Paris, L'Harmattan, coll. «Villes et entreprises », $411 \mathrm{p}$.

—, 2005. - « Organisation de la maîtrise d'ouvrage social, stratégies, objectifs et taches ». Document de travail, ADS, Rabat, 18 p.

PNUD (Programme des Nations Unies pour le Développement) - Unesco, 1985. -Identification des contraintes techniques de réalisation des projets de sauvegarde de Fès. Rapport technique. Paris, 62 p.

—, 1987. Campagne de sawvegarde de Fès. Résultat et recommandations du projet, Rapport final, Paris. 18 p.

\section{Remerciements}

Nous remercions les deux évaluateurs anonymes de la revue Norois, qui ont, par leurs commentaires, amélioré cet article. Le soutien financier de l'Académie Hassan II des Sciences et Techniques (contrat de recherche avec le réseau $R E L O R$ ), la subvention du CNRST accordée au réseau RELOR et le soutien financier apporté au LAGEA par l'Université Sidi Mohamed Ben Abdellah nous sont très utiles.

Cet article a été reçu le 20 janvier 2009 et définitivement accepté le 22 novembre 2009. 
planche I (Brahim AKDIM, Mohamed LAAOUANE - Patrimoine et développement local à Fès...)

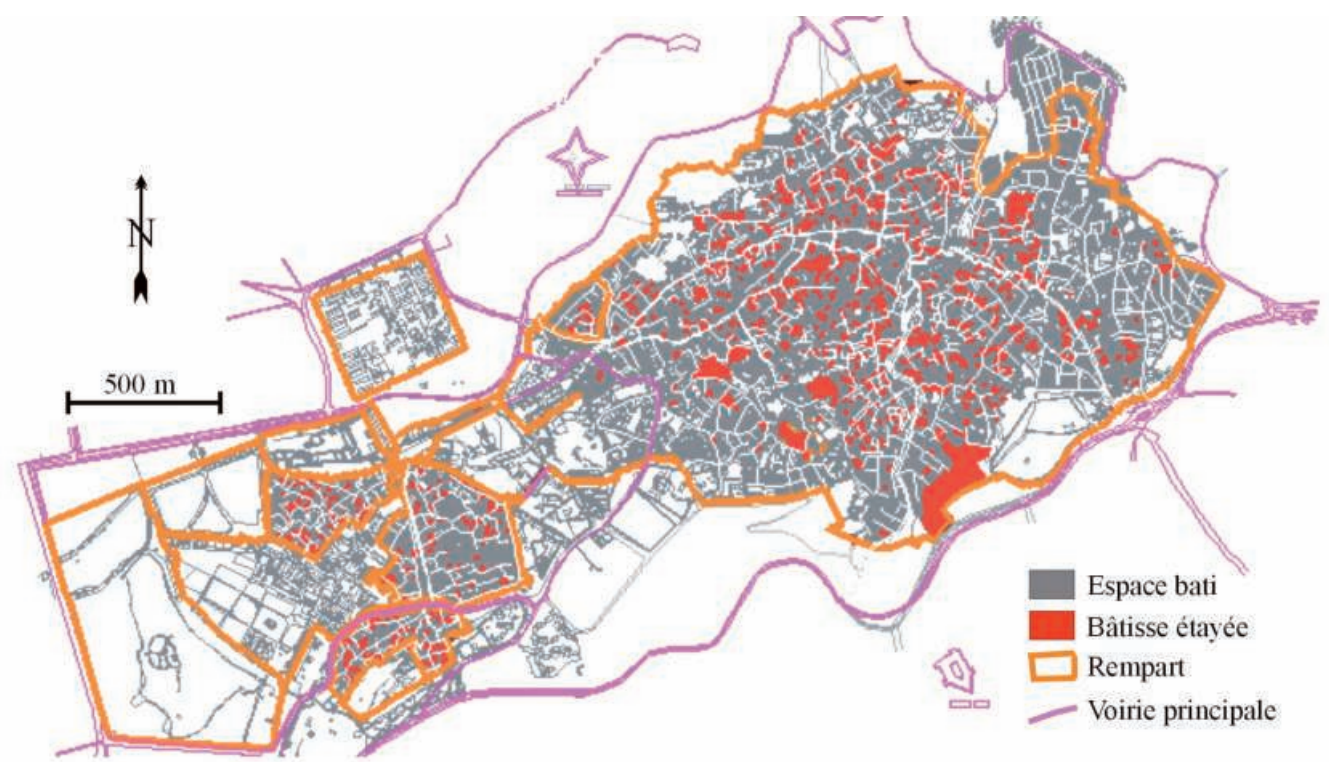

Figure 2 : Sauvegarde du patrimoine habité dans la Médina de Fès : la localisation des bâtisses étayées (source : ADER Fès, d'après El Bouchikhi et al., 2005)

Safeguard of the occupied architectural heritage in the Medina of Fez: the localization of the supported buildings 